\title{
The Effect Of Stellar Pulsation Cycles On Dust Formation: A Temporal Study Of Mid-infrared Spectrum Of O-rich AGB Star, T Cep
}

\author{
Suklima Guha Niyogi and Angela Speck \\ Department of Physics \& Astronomy, University of Missouri, Columbia, MO 65211
}

\begin{abstract}
Pulsation is believed to be the driving mechanism behind mass loss and dust formation around AGB stars. We present a temporal study of T Cep, a long-period Mira variable, which was observed seven times during a 16 month period spanning a single pulsation cycle. The observed spectral dust features change over the pulsation cycle of this Mira. In general, the overall apparent changes in spectral features can be attributed to changes in the dust temperature, resulting from the intrinsic pulsation cycle of the central star. However, not all feature changes are so easily explained. In particular, the classic interpretation of the broad complex features cannot be attributed to a simple mixture of alumina and glassy silicate. The peak features at $9.7,10.8,11.3,13.1 \mu \mathrm{m}$ are better explained by crystalline silicate or mixtures of crystalline silicate and alumina.
\end{abstract}

Keywords: AGB stars - T Cep - pulsation - dust features -crystalline minerals PACS: $97.30 . \mathrm{Jm}$

\section{INTRODUCTION}

We present a temporal study of $\mathrm{T}$ Cep, a long-period Mira of pulsating period of 388 days. T Cep had been classified as spectral type of M5.5e-M8.8e and infrared spectral class of SE1 [1]. It was observed by ISO using SWS seven times in a 16 month period, providing a great opportunity to test hypotheses that link pulsation, stellar variability and dust formation.

\section{OBSERVATION AND ANALYSIS OF T CEP}

We have examined 7 observations of T Cep (which are designated by sequential numbers), stretching from August 1996 to December 1997. These temporally-resolved spectral data were acquired from the Greg Sloan archive 1 . We have also acquired light curve data for T Cep from $\mathrm{AAVSO}^{2}$. The light curve shown in Figure 1, while Table 1 lists the ISO observation dates and visual magnitude for each spectrum.

In order to analyze the dust features around T Cep over a pulsation cycle, we subtracted stellar blackbody from the original data, leaving only the contribution from the dust shell. The stellar emission is approximated by a blackbody of $2500 \mathrm{~K}$. The precise temperature used was determined from the spectral type and its variability

\footnotetext{
${ }^{1} \mathrm{http}: / / \mathrm{isc}$. astro.cornell.edu/ sloan/library/swsatlas/atlas.html

2 http://www.aavso.org/
}

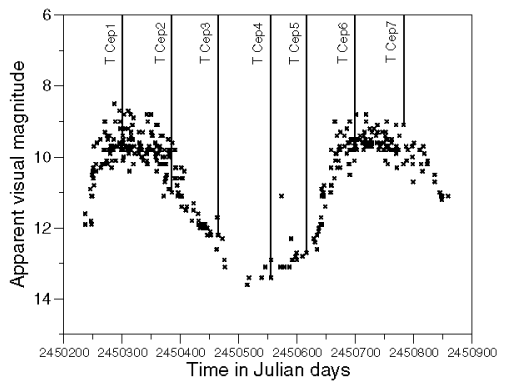

FIGURE 1. Light curve of T Cep over a 16 month period. Plot of apparent visual magnitude over time (in Julian Days). The date of ISO observations are indicated by solid straight lines.

over time (see Table 1). Figure 2 shows plot of flux vs. wavelength over $0-45 \mu \mathrm{m}$ range including the blackbody continuum.

The resulting continuum-subtracted spectra are shown in Figure 3. It is clear from the plot that there is an overall change in strength in the spectral features. But there are four prominent peak features at 9.7, 10.8, 11.3, $13.1 \mu \mathrm{m}$ (shown by straight lines). By analyzing these feature we can constrain the material composition of the dust and other dust properties (e.g. grain-shape, grain-size, crystal structure, dust temperature).

Previous studies showed that the mid-and-far-infrared dust features of O-rich AGB stars provide evidence for the presence of silicate minerals. The broad 9-12 $\mu \mathrm{m}$ feature and the other complex features have been attributed to mixture of glassy silicate and oxides [2]. However, mid-infrared spectra observed by ISO have pro- 
TABLE 1. The observation dates, apparent visual magnitudes and estimated stellar BB temp. for each observations.

\begin{tabular}{lccc}
\hline Obs. & Obs. dates & App vis mag & Temperature of BB $(\mathrm{K})$ \\
\hline T Cep1 & $08 / 05 / 1996$ & 9.4 & 2675 \\
T Cep2 & $10 / 27 / 1996$ & 11.0 & 2511 \\
T Cep3 & $01 / 15 / 1997$ & 12.2 & 2354 \\
T Cep4 & $04 / 13 / 1997$ & 13.4 & 2180 \\
T Cep5 & $06 / 16 / 1997$ & 12.7 & 2316 \\
T Cep6 & $09 / 07 / 1997$ & 9.6 & 2494 \\
T Cep7 & $12 / 01 / 1997$ & 9.1 & 2675 \\
\hline
\end{tabular}

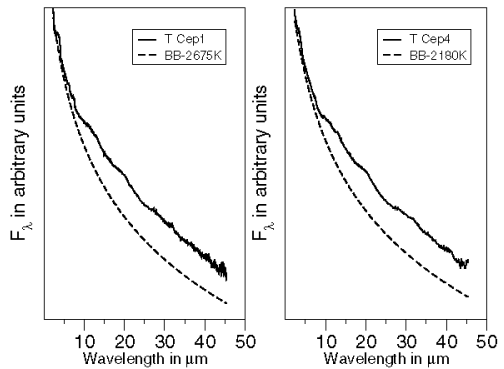

FIGURE 2. Plot of flux (in arbitrary units) vs. wavelength (in $\mu \mathrm{m}$ ). T Cep 1 (solid) is superimposed by blackbody continuum (dashed) of $2675 \mathrm{~K}$ and T Cep4 (solid) is superimposed by blackbody continuum (dashed) of $2180 \mathrm{~K}$.

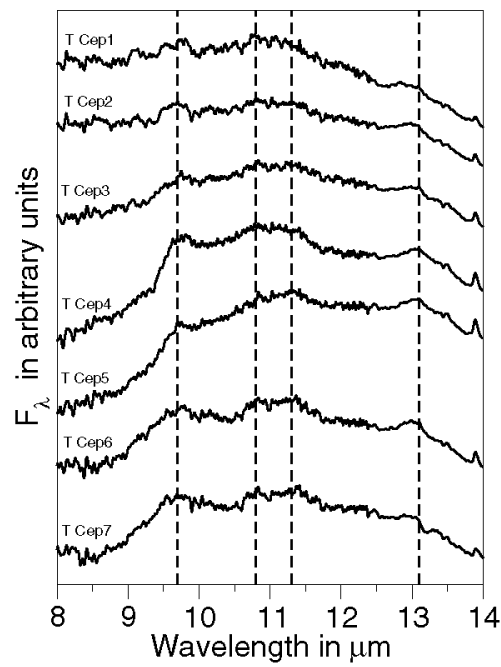

FIGURE 3. Plot of flux vs. wavelength in 8-14 $\mu \mathrm{m}$ region. Blackbody subtracted from original data of each spectrum.

vided striking evidence for the presence of crystalline silicates.[3].
TABLE 2. Combination of flux ratios at peak positions and continuum points along with correlation coefficient $\left(\mathrm{R}^{2}\right)$ among them.

\begin{tabular}{llc}
\hline Flux Ratio & Flux Ratio & Value of $\mathrm{R}^{2}$ \\
\hline $\mathrm{F}_{9.7} / \mathrm{F}_{8.2}$ & $\mathrm{~F}_{10.8} / \mathrm{F}_{8.2}$ & 0.9997 \\
$\mathrm{~F}_{9.7} / \mathrm{F}_{8.2}$ & $\mathrm{~F}_{11.3} / \mathrm{F}_{8.2}$ & 0.999 \\
$\mathrm{~F}_{9.7} / \mathrm{F}_{8.2}$ & $\mathrm{~F}_{13.1} / \mathrm{F}_{8.2}$ & 0.9989 \\
$\mathrm{~F}_{10.8} / \mathrm{F}_{8.2}$ & $\mathrm{~F}_{11.3} / \mathrm{F}_{8.2}$ & 0.9998 \\
$\mathrm{~F}_{10.8} / \mathrm{F}_{8.2}$ & $\mathrm{~F}_{13.1} / \mathrm{F}_{8.2}$ & 0.9997 \\
$\mathrm{~F}_{11.3} / \mathrm{F}_{8.2}$ & $\mathrm{~F}_{13.1} / \mathrm{F}_{8.2}$ & 1.0 \\
$\mathrm{~F}_{9.7} / \mathrm{F}_{25.0}$ & $\mathrm{~F}_{10.8} / \mathrm{F}_{25.0}$ & 0.8692 \\
$\mathrm{~F}_{9.7} / \mathrm{F}_{25.0}$ & $\mathrm{~F}_{11.3} / \mathrm{F}_{25.0}$ & 0.6909 \\
$\mathrm{~F}_{9.7} / \mathrm{F}_{25.0}$ & $\mathrm{~F}_{13.1} / \mathrm{F}_{25.0}$ & $\mathbf{0 . 0 1 3 2}$ \\
$\mathrm{F}_{10.8} / \mathrm{F}_{25.0}$ & $\mathrm{~F}_{11.3} / \mathrm{F}_{25.0}$ & 0.8885 \\
$\mathrm{~F}_{10.8} / \mathrm{F}_{25.0}$ & $\mathrm{~F}_{13.1} / \mathrm{F}_{25.0}$ & $\mathbf{0 . 1 1 5 8}$ \\
$\mathrm{F}_{11.3} / \mathrm{F}_{25.0}$ & $\mathrm{~F}_{13.1} / \mathrm{F}_{25.0}$ & $\mathbf{0 . 3 4 2 1}$ \\
$\mathrm{F}_{9.7} / \mathrm{F}_{10.8}$ & $\mathrm{~F}_{8.2} / \mathrm{F}_{25.0}$ & 0.7023 \\
$\mathrm{~F}_{9.7} / \mathrm{F}_{11.3}$ & $\mathrm{~F}_{8.2} / \mathrm{F}_{25.0}$ & 0.6969 \\
$\mathrm{~F}_{9.7} / \mathrm{F}_{13.1}$ & $\mathrm{~F}_{8.2} / \mathrm{F}_{25.0}$ & 0.8145 \\
$\mathrm{~F}_{10.8} / \mathrm{F}_{11.3}$ & $\mathrm{~F}_{8.2} / \mathrm{F}_{25.0}$ & $\mathbf{0 . 3 9 5 3}$ \\
$\mathrm{F}_{10.8} / \mathrm{F}_{13.1}$ & $\mathrm{~F}_{8.2} / \mathrm{F}_{25.0}$ & 0.728 \\
$\mathrm{~F}_{11.3} / \mathrm{F}_{13.1}$ & $\mathrm{~F}_{8.2} / \mathrm{F}_{25.0}$ & 0.8015 \\
\hline
\end{tabular}

\section{CORRELATION AMONG THE FLUX RATIOS}

To investigate the variability of the dust feature with time, we determine the flux ratios at peak positions $(9.7$, $10.8,11.3,13.1 \mu \mathrm{m}$ ) for each spectra and sought correlation among them. The ratio of the fluxes at continuum points at 8.2 and $25 \mu \mathrm{m}$ has been included as proxy for the continuum temperature (see Table 2).

The overall outcome of the flux ratios is that the features at 9.7 and $11.3 \mu \mathrm{m}$ are strongly correlated with continuum temperature, while the other features at 10.8 and $13.1 \mu \mathrm{m}$ do not correlate (bold entries). The strong correlation with continuum temperature suggests that apparent changes in feature strength are mostly due to the change in dust temperature. However the variability of 10.8 and $13.1 \mu \mathrm{m}$ features suggest that there is an intrinsic change in the dust properties. 


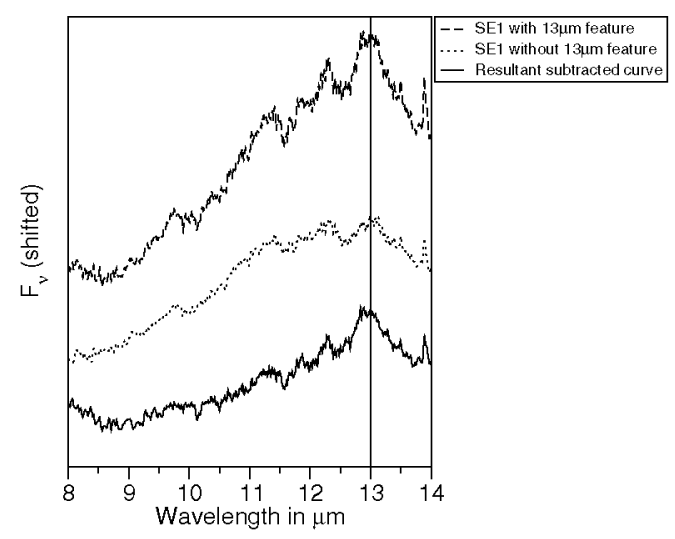

FIGURE 4. Plot of flux vs.wavelength of SE1 class of with (dashed) and without (dotted) $13 \mu \mathrm{m}$ feature. The resultant subtracted plot (solid) shows strong $13 \mu \mathrm{m}$ feature.

\section{MICRON FEATURE}

To investigate the material composition for the $13 \mu \mathrm{m}$ feature, we use the average plot of Silicate Emission (SE1 class) data for with and without $13 \mu \mathrm{m}$, taken from Sloan et al. 2003 [1]. We try to analyze whether this feature could be treated as separate entity from the broad 9-12 $\mu \mathrm{m}$ complex. We simply subtracted SE1 without $13 \mu \mathrm{m}$ feature (dotted) from SE1 with $13 \mu \mathrm{m}$ feature (dashed), shown in Figure 4 plot (solid) has a strong $13 \mu \mathrm{m}$ feature, though the other features are not dominant. Consequently, we assume that the carrier of $13 \mu \mathrm{m}$ feature is not related to the carrier(s) of the other features and previous studies [4]. It is prominent that the subtracted resultant suggests that corundum is a promising carrier for that.

\section{MODEL CALCULATION}

To identify the material composition responsible for the other dust features of T Cep, we compare the observational data with laboratory data of various potential astrominerals. We use optical constants of different minerals to calculate the absorption cross section $\left(\mathrm{C}_{\mathrm{abs}}\right)$ for a spherical particle. We investigate the range of other asymmetrical grain shapes as well. Additionally, we use laboratory data of mass absorption coefficient $(\alpha)$ and absorptivity (A) of different crystalline silicate materials. We use a linear conversion factor to convert calculated $\mathrm{C}_{\mathrm{abs}}$ to $\alpha$ and mix them in different ratios to get the best fit with observations.

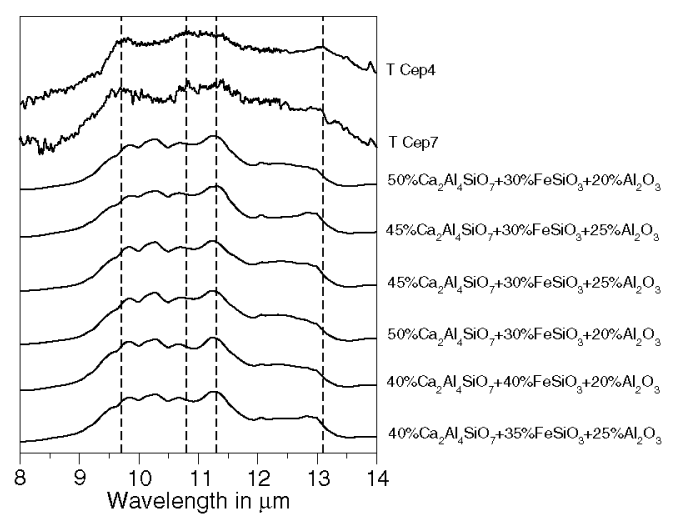

FIGURE 5. Comparison of observed spectra of T Cep with mixture of crystalline silicates and alumina in different proportion.

\section{RESULT}

Figure 5 shows a comparison of observational dust spectra with mixture of different potential crystalline material in $8-14 \mu \mathrm{m}$ region. The top two spectra are observed spectra of $\mathrm{T}$ Cep, and the remaining five are for a mixture of crystalline gehlenite $\left(\mathrm{Ca}_{2} \mathrm{Al}_{4} \mathrm{SiO}_{7}\right)$ taken from Hofmeister et al in (prep.), ferrosilite $\left(\mathrm{FeSiO}_{3}\right)$, taken from Chihara et al. (2002) [5] and corundum [4] $\left(\mathrm{Al}_{2} \mathrm{O}_{3}\right)$ in different proportion. We use laboratory data of $(\alpha)$ for $\left(\mathrm{FeSiO}_{3}\right), \mathrm{A}$ for $\left(\mathrm{Ca}_{2} \mathrm{Al}_{4} \mathrm{SiO}_{7}\right)$ and calculated $\left(\mathrm{C}_{\mathrm{abs}}\right)$ for $\left(\mathrm{Al}_{2} \mathrm{O}_{3}\right)$. A mixture of these crystalline silicates and crystalline alumina fit well with observations.

\section{CONCLUSION}

The overall changes in spectral features with pulsation cycle of $\mathrm{T}$ Cep are mostly due to changing underlying dust continuum temperature. However, the subtle changes in spectral features cannot be explained by dust temperature. The peak features at 9.7, 10.8, 11.3, 13.1 $\mu \mathrm{m}$ can be better explained by mixture of crystalline silicates and corundum, strongly suggesting the presence of crystalline minerals around O-rich AGB stars.

\section{REFERENCES}

1. G. C. Sloan, K. E. Kraemer, J. H. Goebel, and S. D. Price, Astrohys. J. 594, 483-495 (2003).

2. N. J. Woolf, and E. Ney, Astrophys. J. Lett. 155, L181+ (1969).

3. A. K. Speck, M. J. Barlow, R. J. Sylvester, and A. M. Hofmeister, Astron. Astrophys. Suppl. Series 146, 437-464 (2000).

4. K. DePew, A. Speck, and C. Dijkstra, Astrohys. J. 640, 971-981 (2006). 